\title{
PREFAZIONE
}

\section{alla seconda edizione italiana}

La prima edizione di questo manuale apparsa nel 1924 è andata rapidamente esaurendosi; questa e la migliore prova che il libro ebbe favorevole accoglienza. Di buon grado abbiamo accolto l'invito dell'illustre Comm. Dott. Ulrico HoEpLI di por mano alla seconda edizione spinti sopratutto dal desiderio di esprimere il nostro grato animo per la lusinghiera accoglienza accordata alla precedente edizione; non dubitiamo pertanto che la presente sarà altrettanto bene volmente accolta, tanto più che essa, arricchita di nuovi capitoli, è stata aggiornata coll'ultima edizione tedesca.

Con animo riconoscente porgiamo vivi ringraziamenti all'egregio dott. Fernando Straniero per la cortese collaborazione.
L. Soaletta - C. Hornstein. 


\section{PREFAZIONE}

\section{dalla $35^{\text {a }}$ alla $40^{\mathrm{a}}$ edizione tedesca}

In circa quattro anni che sono trascorsi dalla pubblicazione dell'ultima edizione le ricerche sui pesi atomici hanno fatto passi notevoli. Se si confronta la tabella dei pesi atomici recentemente pubblicata per il 1929 con quella allora adottata, risulta che per non meno di 45 elementi, ciò̀ più della. metà di tutti gli elementi con pesi atomici determinati sperimentalmente, sono sopravvenute delte modificazioni. Fra questi elementi è compresa la maggior parte dei valori dei pesi atomici più usati. Da qui la necessità di un'ampia revisione dei calcoli nelle tavole da 1 a 5 ed in questa occasione fu fatto un controllo accurato anche delle parti di queste tavole rimaste invariate. Cosi furono scoperti e corretti dei piccoli errori che erano sfuggiti nelle edizioni precedenti.

Le modificazioni nella tabella dei pesi atomici per quanto copiose, sono di esigua ed in alcuni casi di nessuna importanza nell'applicazione pratica, salvo pochissime eccezioni. Questa particolarità risalterà maggiormente in avvenire, quando le ricerche sui pesi atomici sempre più ampie faciliteranno un'esattezza dell'accertamento di essi, che oltrepassa già i confini della più esatta analisi della pratica normale. Siamo dunque sulla via della stabilità di tutte le quantitd 
vir

costanti praticamente importanti, in opposizione con un prevedibile miglioramento progressivo delle cognizioni esclusivamente scientifiche.

L'indice presenta adesso 25 tavole (compresa la tav. 12a).

Nelle tavole 2 e 3 pud accadere che $i$ multipli presentino piccole differenze rispetto al valore dei numeri semplici (lo stesso per $i$ rispettivi logaritmi). Queste differenze hanno la loro origine nel fatto che ogni peso atomico è moltiplicato integramente e solo il risutato viene arrotondato secondo la regola dell'attendibilità.

Di molto è ampliata la tavola 4. Poichè se ne è vista la necessitd, in seguito a numerose considerazioni sul procedi. mento analitico, prego $i$ consultatori delle tavole di contribuire con ulteriori suggerimenti e desideri, al perfezionamento di questo capitolo. Nella nuova parte B di questa tavola sono state introdotte correzioni per la pesata nell'aria di sostanze per la titolazione; in casi speciali dovrebbero addimostrarsi utili.

Alla tavola 5 è stata data una nuova espressione. Cid̀ significa che questa tavola contiene non solo " fattori " analitici pratici, ma anche quei "fattori " che vengono adoperati per $i$ calcoli stechiometrici.

Nella tavola 9 furono necessarie molte modificazioni a riguardo dei dati recenti sulla densità dei gas, come sono contenuti nella quinta edizione di Landolt-Bornstein. Queste modificazioni si dovettero, naturalmente, apportare anche al contenuto della tavola 10.

Sulla stessa base sono stati corretti $i$ valori della tavola $11 \cdot$ Le indicazioni di parechi autori dimostrano qui in parte divergenze notevoli.

La tavola delle solubilità (16) è stata ampliata e calcolata alla temperatura normale di $20^{\circ}$. Nelle spiegazioni è comunicato quanto ̀̀ necessario sull'origine del valore attribuito. Una 
tabella per la solubilita delle sostanze difficilmente solubili è stata considerata come un futuro desiderio.

La tavola 18 sarà ben accetta non solo al Chimico ed al Fisico, ma anche al Biologo ed al Medico. Per il rimanente si rimanda alle spiegazioni particolari.

L'attuale tavola 19 (Termochimica) riempe una lacuna già lamentata ripetutamente. In essa una trattazione particolare, specialmente della sezione C (calcolo degli equilibri chimici), oltre quella data nelle spiegazioni, non è stata possibile in riguardo allo spazio disponibile; le ulteriori spiegazioni si troveranno nei trattati di chimica-fisica.

La tavola 20 si attiene strettamente alle norme della Commissione per Unità e Simboli, come sono esposte nella raccolta di I. WaLlot: A.E.F. Verhandlungen des Ausschusses Für Einheiten und Formelgrössen in den Iahren 1907 bis 1927. (Berlino. Springer, 1928). Dei segni matematici in essa indicati è stata fatta una scelta di quelli che hanno interesse per il consultatore delle Tavole.

Il contenuto della tavola 24 è stato rettificato ed ampliato in correlazione con $i$ valori recenti (Landolt-Brornstein).

$I$ valori numerici a in essa precedentemente indicati sono stati inclusi nella tavola 23, alla quale propriamente appartenevano già da tempo.

Nell'uso delle mantisse di 5 cifre si riscontreranno casualmente delle piccole discordanze (differenza di un'unitd nell'ultima cifra) nei logaritmi o nei numeri. In casi simili servirà di chiarimento l'intervento di una tavola logaritmica di 7 cifre.

Grande è il numero dei collaboratori volontari, che fin dall'apparire dell'ultima edizione mi hanno aiutato efficacemente con suggerimenti e consigli, con invio di materiale e indicazione di errori. A tutti dico sinceramente grazie.

Anche questa volta devo ringraziare in modo particolare il 
$\mathrm{x}$

Presidente della Commissione Tedesca dei Pesi atomici, Sig. Prof. Dr. O. Hönigschmid di Monaco, che mi mise a cono. scenza delle variazioni delle tabelle dei pesi atomici stabilite per il 1929.

L'esecuzione di una profonda riforma delle tavole, come

è stata necessaria questa volta, è costosa e faticosa, quando si deve mantenere un prezzo di vendita conveniente.

Il pericolo sta in un eccessivo invecchiamento ed è nell'interesse dello stesso consultatore che non consideri le tavole come un libro che si pud lasciare in eredità di padre in figlio, ma bensi, quando appare una nuova edizione, si distacchi dal suo vecchio compagno di lavoro.

Mentre esorto, come ho fatto finora, $i$ collaboratori più lontani ad un perfezionamento sempre migliore del libro, ri. chiamo l'attenzione che la riuscita del collegamento con $i$ mittenti è assicurata solo se il nome e gli appunti sono scritti in modo leggibile.

Marburgo (Lahn), Weissenburgstrasse, 36

Gennaio 1929.

A. Thurt. 